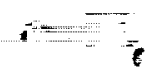

NASA Contractor Report 189682

ICASE Report No. 92-32

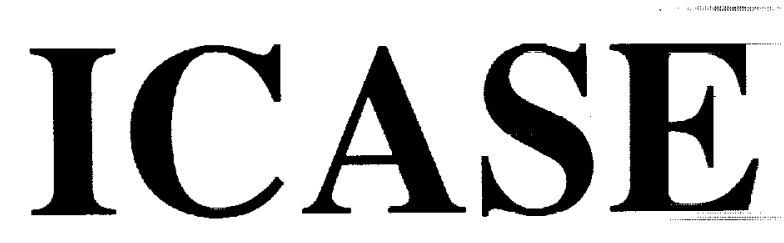

REYNOLDS STRESS CALCULATIONS OF HOMOGENEOUS TURBULENT SHEAR FLOW WITH BOUNDED ENERGY STATES

Charles G. Speziale

R. Abid

Contract Nos. NAS1-18605 and NAS1-19480

July 1992

Institute for Computer Applications in Science and Engineering NASA Langley Research Center

Hampton, Virginia 23665-5225

Operated by the Universities Space Research Association

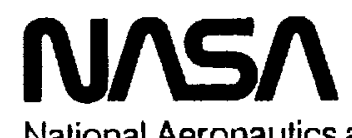

National Aeronautics and

Space Administration

Langley Research Center

Hampton, Virginia 23665-5225

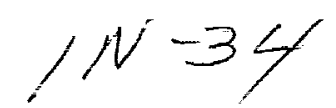

116432

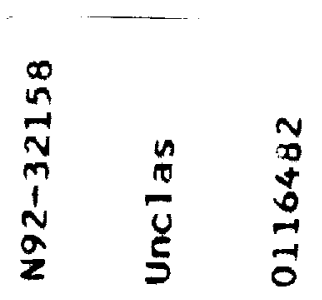

$$
\sum_{0}^{n}
$$

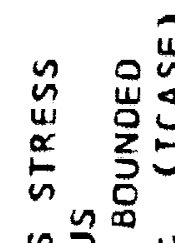

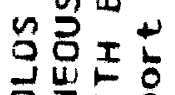

$\frac{1}{2} \sum_{u=0}=0$

$>\frac{4}{\alpha}=0$

u)

온

Nu $\alpha$ u

$\infty 0<4$

o

$\infty z \frac{1}{2}$

$\rightarrow 0$ a

$\frac{1}{x}-2$

$\underset{y}{4}<\frac{z}{4}$

1553

눙

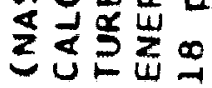




\title{
REYNOLDS STRESS CALCULATIONS OF HOMOGENEOUS TURBULENT SHEAR FLOW WITH BOUNDED ENERGY STATES
}

\author{
Charles G. Speziale* \\ ICASE, NASA Langley Research Center \\ Hampton, VA 23665 \\ R. Abid \\ High Technology Corporation \\ NASA Langley Research Center \\ Hampton, VA 23665
}

\begin{abstract}
Reynolds stress calculations of homogeneous turbulent shear flow are conducted with a second-order closure model modified to account for non-equilibrium vortex stretching in the dissipation rate transport equation as recently proposed by Bernard and Speziale [J. Fluids Engng. 114, 29 (1992)]. As with the earlier reported $K-\varepsilon$ model calculations incorporating this vortex stretching effect, a production-equals-dissipation equilibrium is obtained with bounded turbulent kinetic energy and dissipation. However, this equilibrium is now not achieved until the dimensionless time $S t>60$ - an elapsed time that is at least twice as large as any of those considered in previous numerical and physical experiments on homogeneous shear flow. Direct quantitative comparisons between the model predictions and the results of experiments are quite favorable. In particular, it is shown that the inclusion of this nonequilibrium vortex stretching effect has the capability of explaining the significant range of production to dissipation ratios observed in experiments.
\end{abstract}

\footnotetext{
*This research was supported by the National Aeronautics and Space Administration under NASA Contract No. NAS1-18605 and NAS1-19480 while the first author was in residence at the Institute for Computer Applications in Science and Engineering (ICASE), NASA Langley Research Center, Hampton, VA 23665.
} 


\section{INTRODUCTION}

In transport models for the turbulent dissipation rate it is traditionally assumed that an equilibrium exists where the production of dissipation by vortex stretching is exactly counterbalanced by the leading order part of the destruction of dissipation term (see Tennekes and Lumley 1972 and Lesieur 1990). Recently, Bernard and Speziale (1992) proposed a new transport model for the dissipation rate with residual vortex stretching to account for small departures from equilibrium. This new model - which is of the same general form as that obtained from the theory of self-preservation (see Speziale and Bernard 1992 and George 1992) - was shown to yield a more complete description of isotropic decay. More importantly, however, it was demonstrated by Bernard and Speziale (1992) that the inclusion of this vortex stretching effect yields a production-equals-dissipation equilibrium in homogeneous shear flow, with bounded turbulent kinetic energy and dissipation. Prior to saturation which occurs at an elapsed time larger than any of those considered in previously conducted physical and numerical experiments - the turbulent kinetic energy and dissipation rate grow exponentially with time. Good qualitative agreement between the model predictions with vortex stretching, and the results of physical and numerical experiments on homogeneous shear flow, was demonstrated by Bernard and Speziale (1992) and Bernard, Thangam and Speziale (1992). However, they were not able to make more detailed quantitative comparisons with experiments since, for simplicity, they based their calculations on the $K-\varepsilon$ model. This establishes the motivation of the present paper: to present full Reynolds stress calculations of homogeneous shear flow with a state of the art second-order closure model that incorporates this vortex stretching effect.

In this paper, more detailed calculations of homogeneous shear flow are presented based on the second-order closure model of Speziale, Sarkar and Gatski (1991) modified to account for non-equilibrium vortex stretching in the dissipation rate transport equation. For elapsed times $S t<30$, vortex stretching has little qualitative effect on the solution which exhibits an exponential time growth of turbulent kinetic energy and dissipation that is in good agreement with the results of physical and numerical experiments (see Tavoularis and Karnik 1989 and Rogers, Moin and Reynolds 1986). However, for $S t>60$ (which is far beyond the largest elapsed time that has been considered in experiments), the flow saturates to a productionequals-dissipation equilibrium. Interesting features of this vortex stretching solution - which include its apparent ability to explain the wide range of production to dissipation ratios observed in experiments - are discussed along with the implications for turbulence modeling. 


\section{FORMULATION OF THE PROBLEM}

We consider homogeneous turbulent shear flow where the mean velocity gradient tensor takes the form

$$
\frac{\partial \bar{u}_{i}}{\partial x_{j}}=S \delta_{i 1} \delta_{j 2}
$$

given that $S$ is a constant shear rate and $\delta_{i j}$ is the Kronecker delta. In the usual formulations of homogeneous shear flow, an initially decaying isotropic turbulence is subjected to the uniform shear rate $S$ at time $t=0$. The Reynolds stress tensor $\tau_{i j} \equiv \overline{u_{i}^{\prime} u_{j}^{\prime}}$ is a solution of the transport equation (c.f. Hinze 1975)

$$
\dot{\tau}_{i j}=-\tau_{i k} \frac{\partial \bar{u}_{j}}{\partial x_{k}}-\tau_{j k} \frac{\partial \bar{u}_{i}}{\partial x_{k}}+\Pi_{i j}-\frac{2}{3} \varepsilon \delta_{i j}
$$

in incompressible homogeneous turbulence, where a superposed dot represents a time derivative and

$$
\Pi_{i j} \equiv \overline{p^{\prime}\left(\frac{\partial u_{i}^{\prime}}{\partial x_{j}}+\frac{\partial u_{j}^{\prime}}{\partial x_{i}}\right)}, \quad \varepsilon=\nu \overline{\frac{\partial u_{i}^{\prime}}{\partial x_{j}} \frac{\partial u_{i}^{\prime}}{\partial x_{j}}}
$$

denote, respectively, the pressure-strain correlation and the turbulent dissipation rate. In (3), $p^{\prime}$ and $u_{i}^{\prime}$ represent the fluctuating pressure and fluctuating velocity, respectively, and $\nu$ denotes the kinematic viscosity of the fluid; as with most model studies of high-Reynoldsnumber turbulence, Kolmogorov's assumption of local isotropy is invoked for the dissipation rate.

In order to achieve closure, models must be provided for the pressure-strain correlation $\Pi_{i j}$ and the turbulent dissipation rate $\varepsilon$. The recent pressure-strain model of Speziale, Sarkar and Gatski (1991) is utilized which takes the form:

$$
\begin{aligned}
\Pi_{i j}= & -\left(C_{1} \varepsilon+C_{1}^{*} \mathcal{P}\right) b_{i j}+C_{2} \varepsilon\left(b_{i k} b_{k j}-\frac{1}{3} b_{k l} b_{k l} \delta_{i j}\right) \\
& +\left(C_{3}-C_{3}^{*} I I^{1 / 2}\right) K \bar{S}_{i j}+C_{4} K\left(b_{i k} \bar{S}_{j k}+b_{j k} \bar{S}_{i k}\right. \\
& \left.-\frac{2}{3} b_{k l} \bar{S}_{k l} \delta_{i j}\right)+C_{5} K\left(b_{i k} \bar{W}_{j k}+b_{j k} \bar{W}_{i k}\right)
\end{aligned}
$$

where

$$
\begin{gathered}
b_{i j}=\left(\tau_{i j}-\frac{2}{3} K \delta_{i j}\right) / 2 K, \quad K=\frac{1}{2} \tau_{i i} \\
I I=b_{i j} b_{i j}, \quad \mathcal{P}=-\tau_{i j} \frac{\partial \bar{u}_{i}}{\partial x_{j}} \\
\bar{S}_{i j}=\frac{1}{2}\left(\frac{\partial \bar{u}_{i}}{\partial x_{j}}+\frac{\partial \bar{u}_{j}}{\partial x_{i}}\right), \quad \bar{W}_{i j}=\frac{1}{2}\left(\frac{\partial \bar{u}_{i}}{\partial x_{j}}-\frac{\partial \bar{u}_{j}}{\partial x_{i}}\right)
\end{gathered}
$$


given that $b_{i j}$ is the anisotropy tensor, $K$ is the turbulent kinetic energy, and $\mathcal{P}$ is the turbulence production. The constants of the model are given as follows: $C_{1}=3.4, C_{1}^{*}=1.80$, $C_{2}=4.2, C_{3}=4 / 5, C_{3}^{*}=1.30, C_{4}=1.25$ and $C_{5}=0.40$. Equation (2) is solved with the modeled dissipation rate transport equation

$$
\dot{\varepsilon}=C_{\varepsilon 1} \frac{\varepsilon}{K} \mathcal{P}+C_{\varepsilon 3} R_{t}^{1 / 2} \frac{\varepsilon^{2}}{K}-C_{\varepsilon 2} \frac{\varepsilon^{2}}{K}
$$

where $R_{t} \equiv K^{2} / \nu \varepsilon$ is the turbulence Reynolds number and $C_{\varepsilon 1}, C_{\varepsilon 2}$ and $C_{\varepsilon 3}$ are constants (see Bernard and Speziale 1992). The term containing $C_{\varepsilon 3}$ occurs when there is a departure from equilibrium so that there is an imbalance between the production of dissipation by vortex stretching and the leading order part of the destruction of dissipation term which each scale as $R_{t}^{1 / 2}$. Equation (8) is of the same general mathematical form as that obtained from the theory of self-preservation (see Speziale and Bernard 1992 and George 1992). The standard modeled dissipation rate transport equation is recovered in the limit as $C_{\varepsilon 3}$ goes to zero. The same values of $C_{\epsilon 1}$ and $C_{\varepsilon 2}$ as proposed in the Speziale, Sarkar and Gatski (SSG) model are chosen:

$$
C_{\varepsilon 1}=1.44, \quad C_{\varepsilon 2}=1.83
$$

A variety of values of $C_{\varepsilon 3}$ in the range of 0.001 to 0.01 will be considered (these represent small imbalances in vortex stretching of the order of $0.1 \%-1.0 \%$; of course, the magnitude of this imbalance can depend on the initial conditions).

The governing nonlinear differential equations for homogeneous shear flow are obtained by substituting (1) into (2) and (8) while making use of the pressure-strain model (4). Five coupled nonlinear differential equations for $\tau_{11}, \tau_{12}, \tau_{22}, \tau_{33}$, and $\varepsilon$ comprise this system. These coupled equations can be easily converted to an equivalent set in terms of $b_{11}, b_{12}, b_{22}, b_{33}$ and $S K / \varepsilon$ which constitute the non-dimensional structural parameters of the problem that achieve equilibrium values that are independent of the initial conditions (see Speziale and Mac Giolla Mhuiris 1989a,b). The governing system of differential equations are solved subject to the initial conditions:

$$
b_{i j}=0, \quad R_{t}=R_{t_{0}}, \quad \frac{S K}{\varepsilon}=\frac{S K_{0}}{\varepsilon_{0}}
$$

at time $t=0$. Only the solutions with net vortex stretching $\left(C_{\varepsilon 3}>0\right)$ require the specification of the initial turbulence Reynolds number.

\section{DISCUSSION OF THE RESULTS}

We now present results obtained from a Runge-Kutta numerical integration scheme. In Figure $1(\mathrm{a})$, the time evolution of the dimensionless turbulent kinetic energy $\left(K^{*} \equiv K / K_{0}\right)$ 
is shown as a function of the dimensionless time $\left(t^{*} \equiv S t\right)$ for $S K_{0} / \varepsilon_{0}=3.38$ and $R_{t_{0}}=750$ the initial conditions of the large-eddy simulation of Bardina, Ferziger and Reynolds (1983). Here, we set $C_{\varepsilon 3}=0.001$ so that there is only a minute imbalance in vortex stretching of the order of $0.1 \%$. As with the earlier reported calculations with the SSG model where $C_{\varepsilon 3}=0$, there is excellent agreement between the model predictions and the large-eddy simulation results as illustrated in Figure 1(a). However, while there is virtually no difference between the short-time predictions of the SSG model with $C_{\varepsilon 3}=0$ and $C_{\varepsilon 3}=0.001$, there is a distinct difference in the long-time solutions. When $C_{\varepsilon 3}=0$, the kinetic energy and dissipation rate grow unbounded with time; for $t^{*} \gg 1$,

$$
K^{*} \sim e^{\lambda t^{*}}, \varepsilon^{*} \sim e^{\lambda t^{*}}
$$

so that $K^{*}$ and $\varepsilon^{*} \rightarrow \infty$ as $t^{*} \rightarrow \infty$. For any finite $C_{\varepsilon 3}-$ no matter how small it may be the turbulent kinetic energy and dissipation rate eventually saturate to bounded equilibrium values after an early time exponential growth as shown in Figure 1(b).

In Figure 2, the time evolution of the turbulent kinetic energy is shown for the same initial conditions $\left(S K_{0} / \varepsilon_{0}=3.38, R_{t_{0}}=750\right)$ but with a variety of different values for $C_{\varepsilon 3}$. It is clear from these results that the value of $C_{\varepsilon 3}$ determines the saturation level of the turbulent kinetic energy. For sufficiently small $C_{\varepsilon 3}$, the turbulent kinetic energy grows exponentially for $S t<30$ - the largest elapsed time considered in all previously conducted physical and numerical experiments. Here, saturation does not occur until $S t>60$, which is a value that is more than double that of any considered in previous experiments. If the vortex stretching solution were examined in isolation for elapsed times $S t<30$, it could be erroneously concluded that there is an unbounded exponential time growth of turbulent kinetic energy. This points to the danger of drawing conclusions about long-time asymptotic. states from data corresponding to only limited elapsed times.

In Figure 3(a), the time evolution of the ratio of production to dissipation $\mathcal{P} / \varepsilon$ predicted by the SSG model both with and without vortex stretching is shown. The non-zero values of the constant $C_{\varepsilon 3}$ are in the range of 0.001-0.009 (an imbalance in vortex stretching of the order of $0.1 \%-1 \%$ ). It is clear from this figure that just a minute imbalance in vortex stretcling can cause a significant spread in $\mathcal{P} / \varepsilon$ at $S t=30$. This is reminiscent of the experiments of Tavoularis and Karnik (1989) where, for comparably large shear rates $S K_{0} / \varepsilon_{0}>1$, values of $\mathcal{P} / \varepsilon$ in the range of $1.4-1.8$ have been observed at the latest station measured which corresponds to $S t \leq 30$. Furthermore, $\mathcal{P} / \varepsilon$ peaks at $S t \approx 20$ before asymptoting to a value of $\mathcal{P} / \varepsilon=1$ which is achieved for values of $S t>60$ as shown in Figure 3(b). This phenomenon of a local maximum being reached, followed a gradual dropoff in the time interval $0 \leq S t \leq 30$, is reminiscent of results observed in physical and numerical experiments (see the direct 
simulations of Rogers et al. 1986 shown in Figure 4). Without this vortex stretching effect, second-order closures predict that $\mathcal{P} / \varepsilon$ goes to an equilibrium value of $\left(C_{\varepsilon 2}-1\right) /\left(C_{\varepsilon 1}-1\right)$ monotonically, getting close by the time $S t=20$.

As in the absence of this vortex stretching effect, second-order closure models predict that $b_{i j}$ and $S K / \varepsilon$ achieve equilibrium values that are independent of the initial conditions as well as the constants $C_{\varepsilon 1}, C_{\varepsilon 2}$ and $C_{\varepsilon 3}$. In Table 1, the equilibrium values predicted by the standard SSG model $\left(C_{\varepsilon 3}=0\right)$ and the SSG model with vortex stretching $\left(C_{\varepsilon 3}>\right.$ 0 ) are compared with the physical and numerical experiments of Tavoularis and Karnik (1989) and Rogers et al. (1986). The inclusion of vortex stretching only causes modest deviations from the experimental values for the anisotropies; only the shear parameter is affected substantially. However, it must be remembered that the physical and numerical experiments have only been conducted for $S t \leq 30$. For the case where $S K_{0} / \varepsilon_{0}=3.38$, $R_{t_{0}}=750$ and $C_{\varepsilon 3}=0.001$ shown in Figure 1, the SSG model with vortex stretching predicts that

$$
b_{11}=0.215, b_{12}=-0.163, b_{22}=-0.141, b_{33}=-0.074, S K / \varepsilon=5.40
$$

at $S t=30$ - results that are in close proximity to the experimental data which is given on Table 1 .

\section{CONCLUDING REMARKS}

The Reynolds stress calculations presented in this study clearly indicate that the introduction of the non-equilibrium vortex stretching effect of Bernard and Speziale (1992) in a recently proposed second-order closure model does not compromise the accuracy of its quantitative predictions for homogeneous shear flow. In fact, when the SSG model was modified to incorporate this vortex stretching effect, better agreement with experimental data was achieved on two fronts: (a) consistent with experiments, a universal equilibrium was not reached by $S t=30$, and (b) a variety of production to dissipation ratios in the range $1<\mathcal{P} / \varepsilon<1.8$, depending on the initial conditions, were obtained at $S t=30$ consistent with experiments $(\mathcal{P} / \varepsilon$ asymptotes to 1 for elapsed times $S t>60)$. In our opinion, the level of agreement between the model predictions and experiments is of such quality that a production-equals-dissipation equilibrium, with bounded energy states, cannot be ruled out as a viable alternative to the classically accepted hypothesis of unbounded energy growth.

As discussed by Bernard and Speziale (1992), the question of whether or not homogeneous shear flow actually saturates to a production-equals-dissipation equilibrium remains an open question that will only be firmly resolved by a rigorous mathematical analysis. However, even if it were to be ultimately proved that homogeneous shear flow has unbounded energy growth, 
this would be due to the idealized nature of the problem; real physical systems saturate before singularities occur. The introduction of this vortex stretching effect in Reynolds stress closures could lead to better behaved models by preventing such singularities, without compromising their ability to collapse experimental data in benchmark turbulent flows like homogeneous shear flow.

\section{ACKNOWLEDGEMENT}

The second author (RA) would like to acknowledge the support of NASA Langley Research Center through a contract to High Technology Corporation. 


\section{REFERENCES}

Bardina, J., Ferziger, J. H. and Reynolds, W. C., 1983. "Improved Turbulence Models Based on Large-Eddy Simulation of Homogeneous, Incompressible Turbulent Flows," Stanford University Technical Report TF-19.

Bernard, P. S. and Speziale, C. G., 1992. "Bounded Energy States in Homogeneous Turbulent Shear Flow - An Alternative View," J. Fluids Engng., Vol. 114, pp. 29-39.

Bernard, P. S., Thangam, S. and Speziale, C. G., 1992. "The Role of Vortex Stretching in Turbulence Modeling," ICASE/LaRC Series on Transition and Turbulence, SpringerVerlag (to appear).

George, W. K., 1992. "The Decay of Homogeneous Isotropic Turbulence," Phys. Fluids A, Vol. 4, pp. 1492-1509.

Hinze, J. O., 1975. Turbulence, McGraw-Hill, NY.

Lesieur, M., 1990. Turbulence in Fluids, 2nd Edition, Martinus Nijhoff, Boston, MA.

Rogers, M. M., Moin, P. and Reynolds, W. C., 1986, "The Structure and Modeling of the Hydrodynamic and Passive Scalar Fields in Homogeneous Turbulent Shear Flow," Stanford University Technical Report TF-25.

Speziale, C. G. and Bernard, P. S., 1992. "The Energy Decay in Self-Preserving Isotropic Turbulence Revisited," J. Fluid Mech., Vol. 241, pp. 645-667.

Speziale, C. G. and Mac Giolla Mhuiris, N., 1989a. "On the Prediction of Equilibrium States in Homogeneous Turbulence," J. Fluid Mech., Vol. 209, pp. 591-615.

Speziale, C. G. and Mac Giolla Mhuiris, N., 1989b. "Scaling Laws for Homogeneous Turbulent Shear Flows in a Rotating Frame," Phys. Fluids A, Vol. 1, pp. 294-301.

Speziale, C. G., Sarkar, S. and Gatski, T. B., 1991. "Modeling the Pressure-Strain Correlation of Turbulence: An Invariant Dynamical Systems Approach," J. Fluid Mech., Vol. 227, pp. 245-272.

Tavoularis, S. and Karnik, U., 1989. "Further Experiments on the Evolution of Turbulent Stresses and Scales in Uniformly Sheared Turbulence," J. Fluid Mech., Vol. 204, pp. $457-478$. 
Tennekes, H. and Lumley, J. L., 1972. A First Course in Turbulence, M.I.T. Press, Cambridge, MA. 


\begin{tabular}{|c|c|c|c|}
\hline $\begin{array}{c}\text { Equilibrium } \\
\text { Values }\end{array}$ & $\begin{array}{c}\text { SSG Model } \\
\left(C_{\varepsilon 3}=0\right)\end{array}$ & $\begin{array}{c}\text { SSG Model } \\
\left(C_{\varepsilon 3}>0\right)\end{array}$ & $\begin{array}{c}\text { Experimental } \\
\text { Data }\end{array}$ \\
\hline$b_{11}$ & 0.219 & 0.201 & 0.21 \\
\hline$b_{22}$ & -0.146 & -0.127 & -0.14 \\
\hline$b_{33}$ & -0.073 & -0.074 & -0.07 \\
\hline$b_{12}$ & -0.164 & -0.160 & -0.16 \\
\hline$S K / \varepsilon$ & & & 5.0 \\
\hline
\end{tabular}

इ Table 1. Comparison of the predictions of the SSG model for the equilibrium values in homogeneous shear flow with physical and numerical experiments (Tavoularis and Karnik 1989 and Rogers et al. 1986). 
(a)

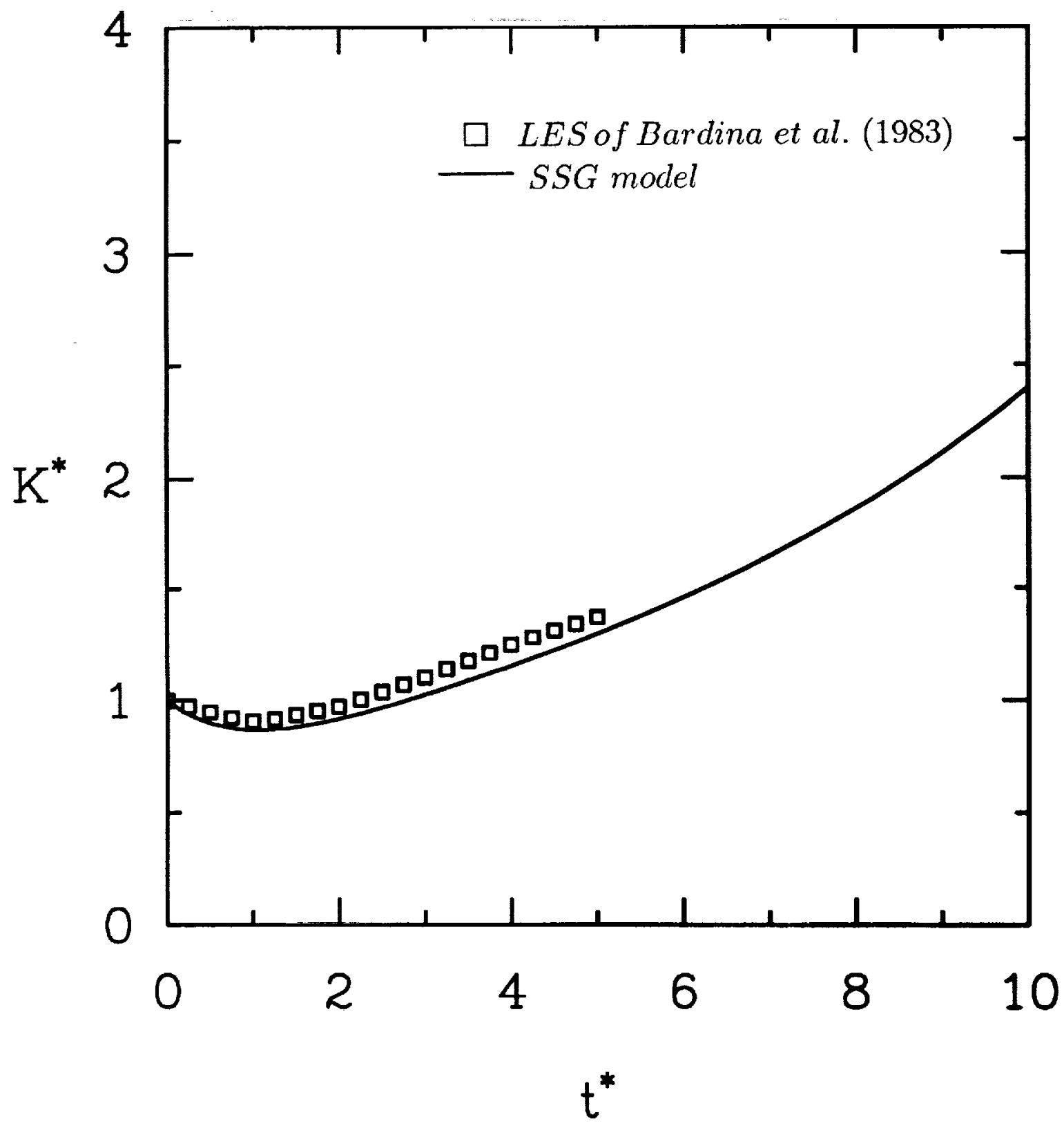

Figure 1. Time evolution of the turbulent kinetic energy in homogeneous shear flow: $S K_{0} / \varepsilon_{0}$ $=3.38$ and $R_{t_{0}}=750$. (a) Comparison of the vortex-stretching modified SSG model $\left(C_{\varepsilon 3}=\right.$ 0.001) with the large-eddy simulation of Bardina et al. (1983), and (b) the long-time SSG model predictions $\left(C_{e 3}=0\right.$ and $\left.C_{\varepsilon 3}=0.001\right)$. 
(b)

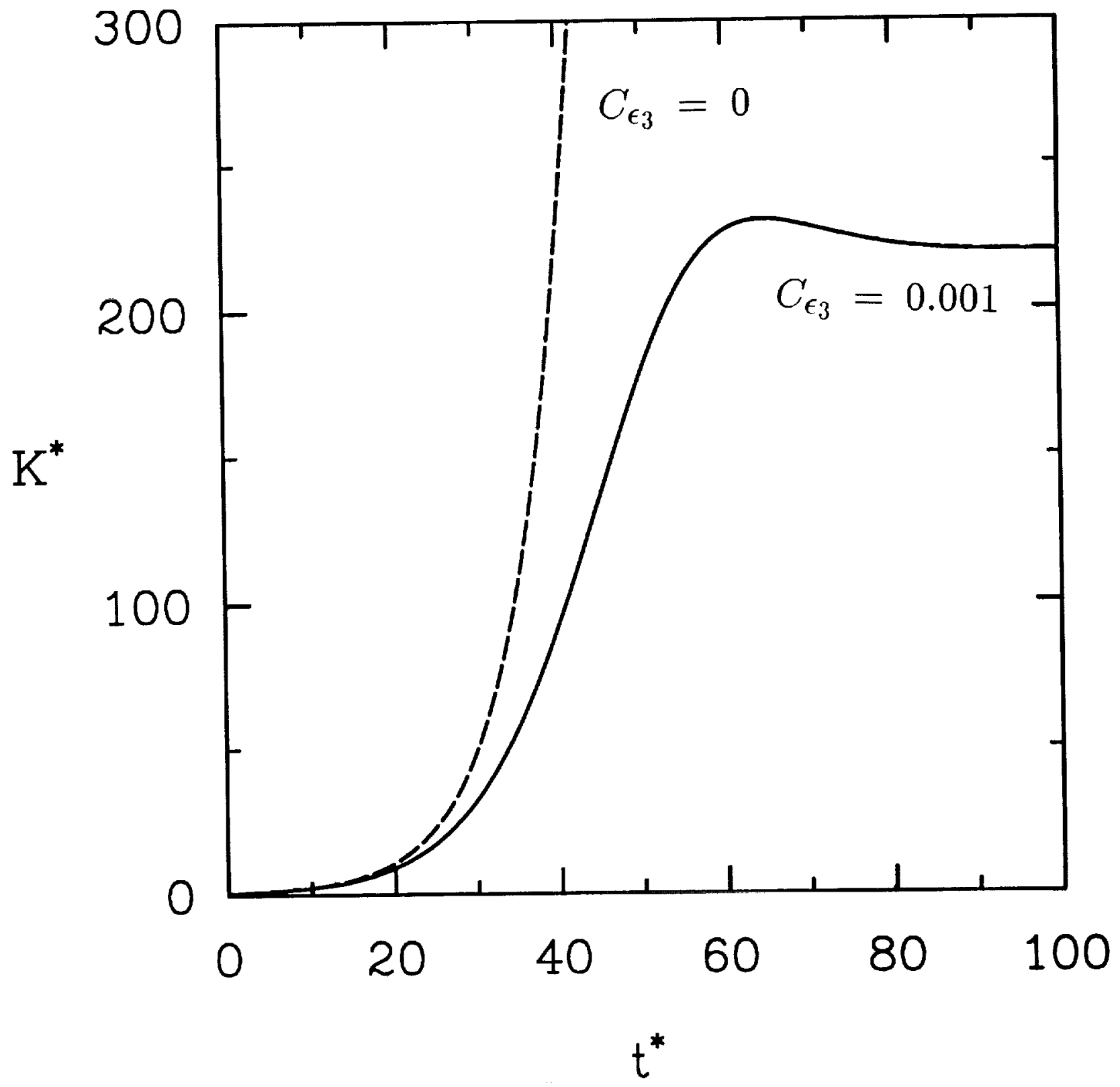

Figure 1. Time evolution of the turbulent kinetic energy in homogeneous shear flow: $S K_{0} / \varepsilon_{0}$ $=3.38$ and $R_{t_{0}}=750$. (a) Comparison of the vortex-stretching modified SSG model $\left(C_{\varepsilon 3}=\right.$ 0.001) with the large-eddy simulation of Bardina et al. (1983), and (b) the long-time SSG model predictions $\left(C_{\varepsilon 3}=0\right.$ and $\left.C_{\varepsilon 3}=0.001\right)$. 


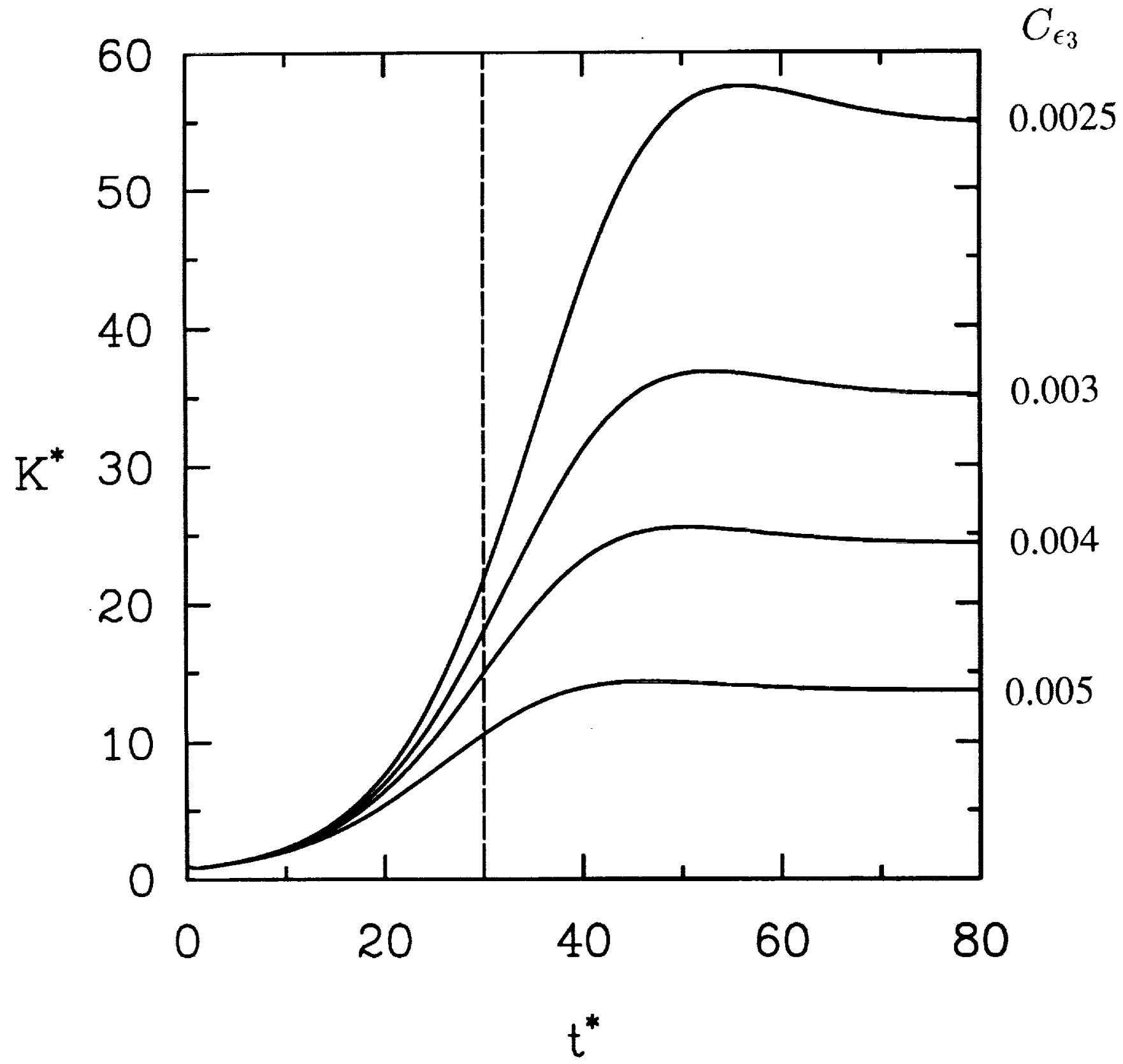

Figure 2. Time evolution of the turbulent kinetic energy predicted by the SSG model with vortex stretching for a range of values of $C_{\varepsilon 3} ; S K_{0} / \varepsilon_{0}=3.38$ and $R_{t_{0}}=750$. 


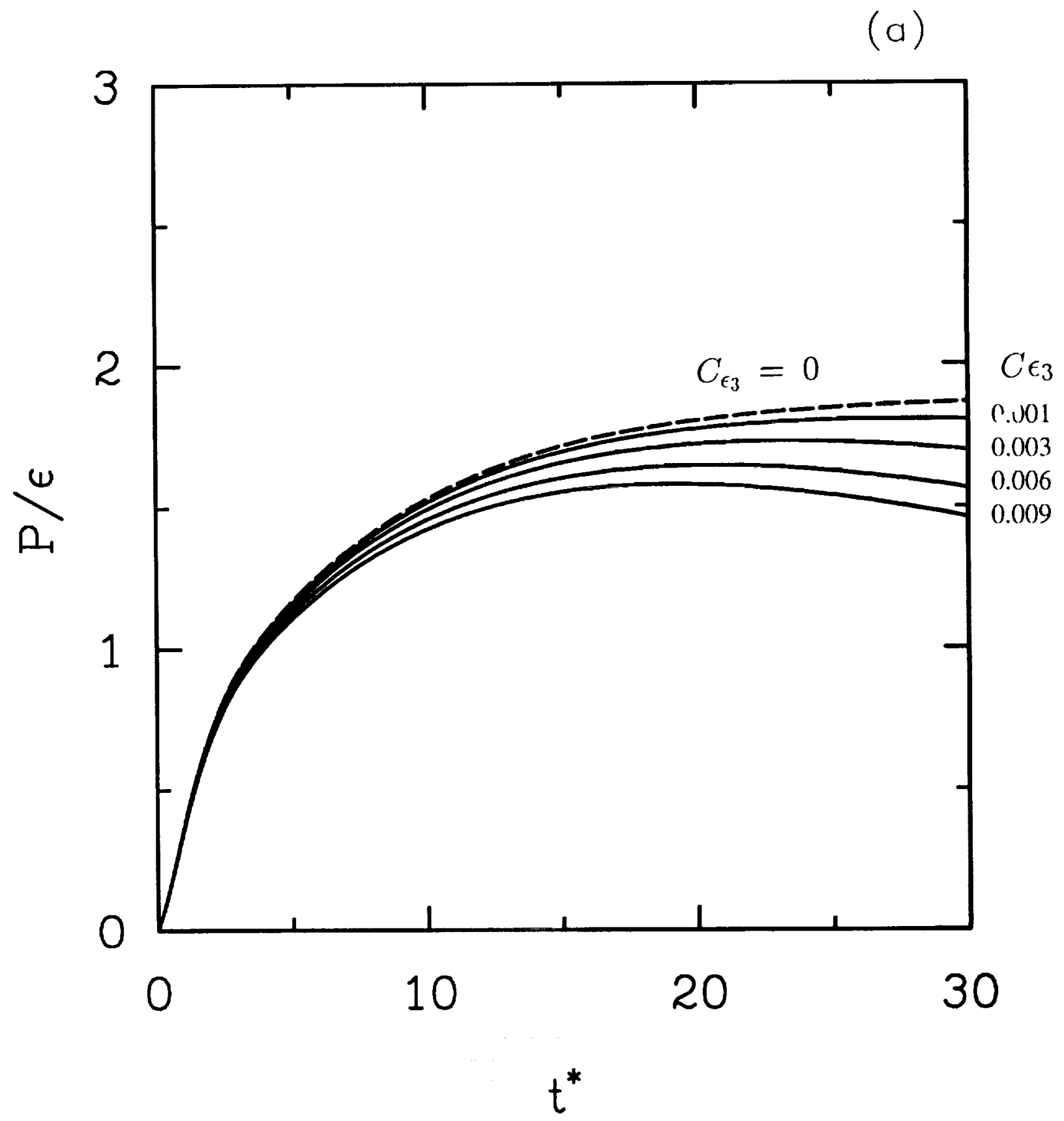

Figure 3. Time evolution of the ratio of production to dissipation in homogeneous shear flow for $S K_{0} / \varepsilon_{0}=3.38$ and $R_{t_{0}}=750$ : (a) Predictions of the $S S G$ model for a variety of values of $C_{\varepsilon 3}$ for $S t \leq 30$, and (b) Predictions of the SSG model for $S t \leq 100$. 
(b)

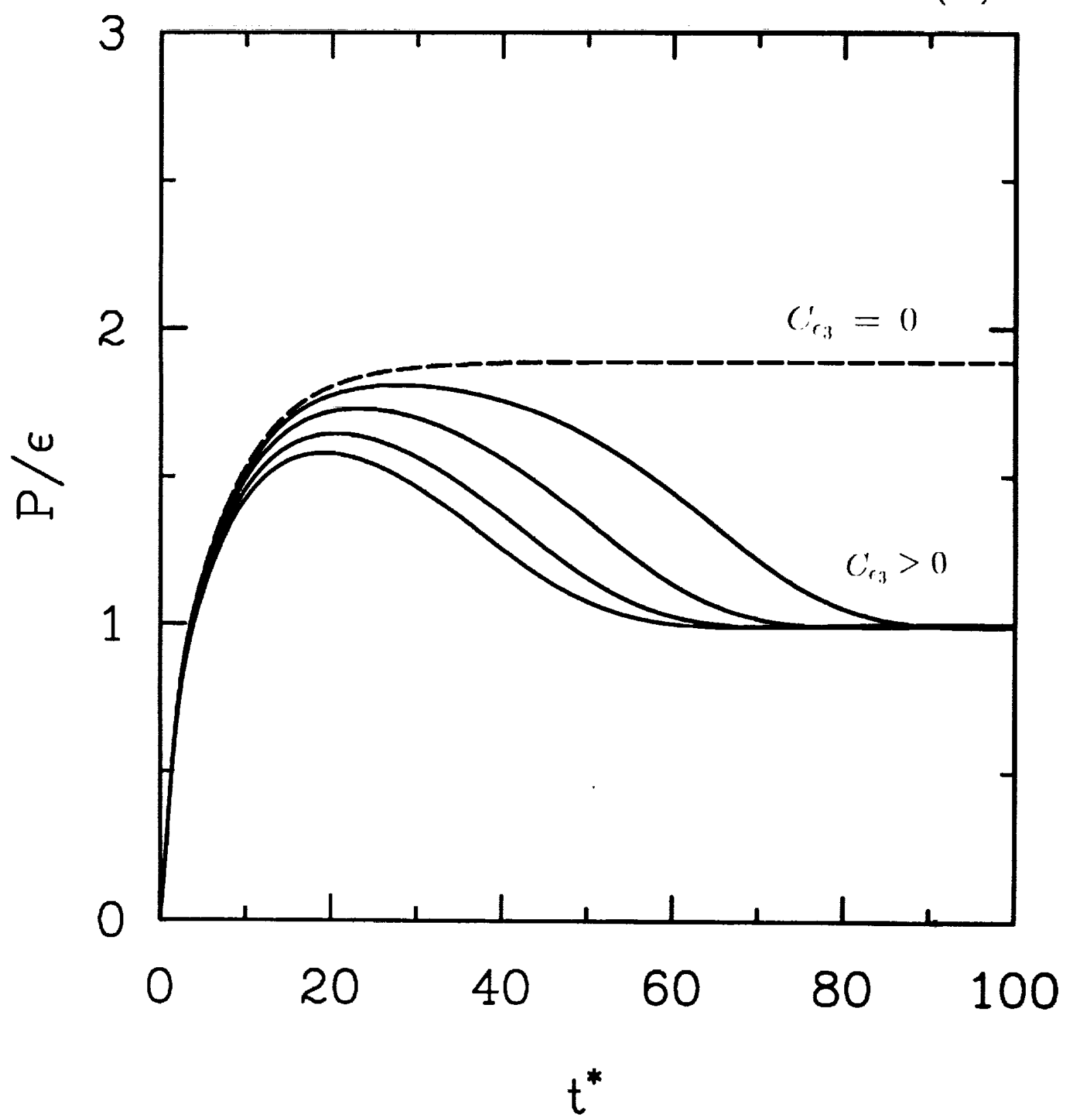

Figure 3. Time evolution of the ratio of production to dissipation in homogeneous shear flow for $S K_{0} / \varepsilon_{0}=3.38$ and $R_{t_{0}}=750$ : (a) Predictions of the SSG model for a variety of values of $C_{\varepsilon 3}$ for $S t \leq 30$, and (b) Predictions of the SSG model for $S t \leq 100$. 


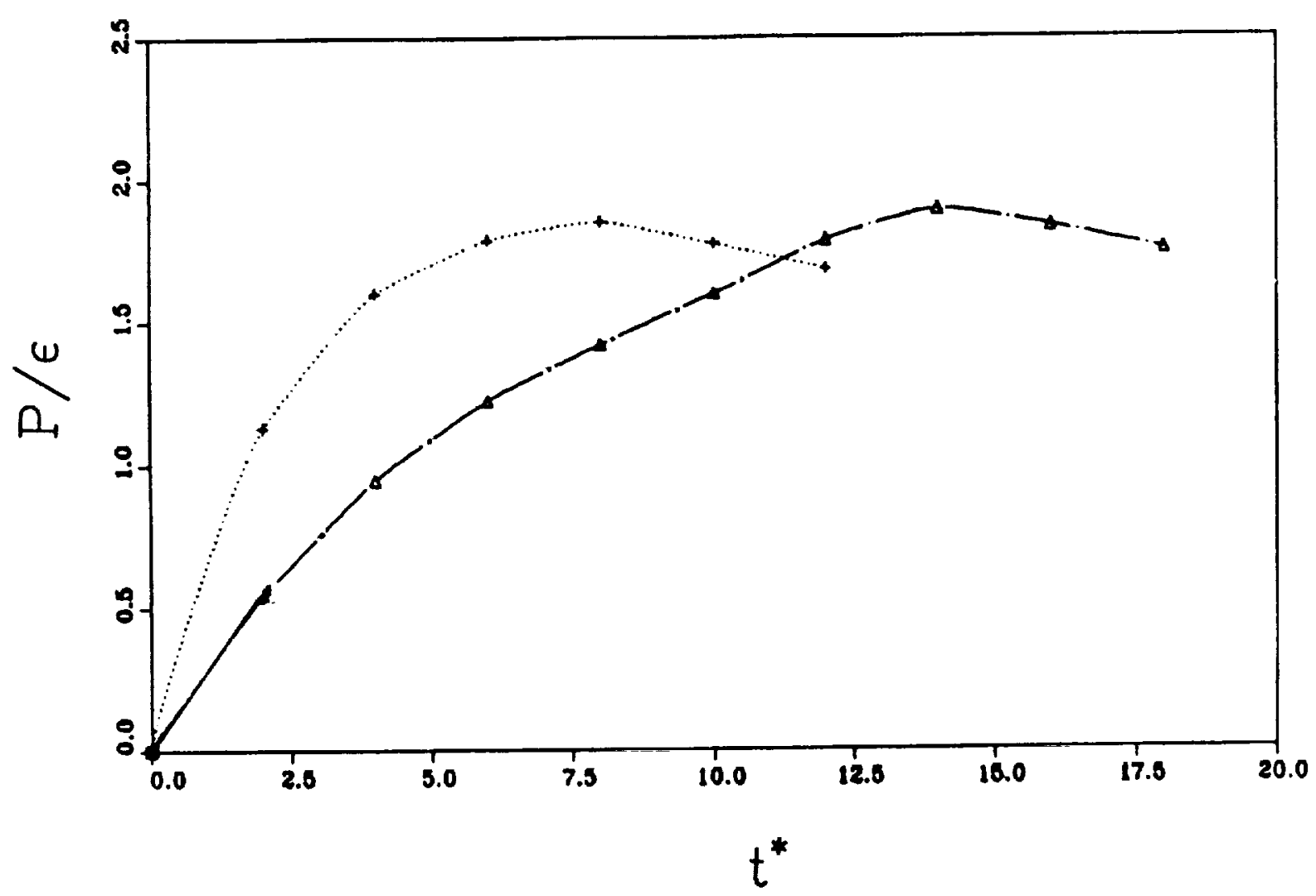

Figure 4. Time evolution of the ratio of production to dissipation taken from the direct numerical simulations of homogeneous shear flow by Rogers et al. (1986). 


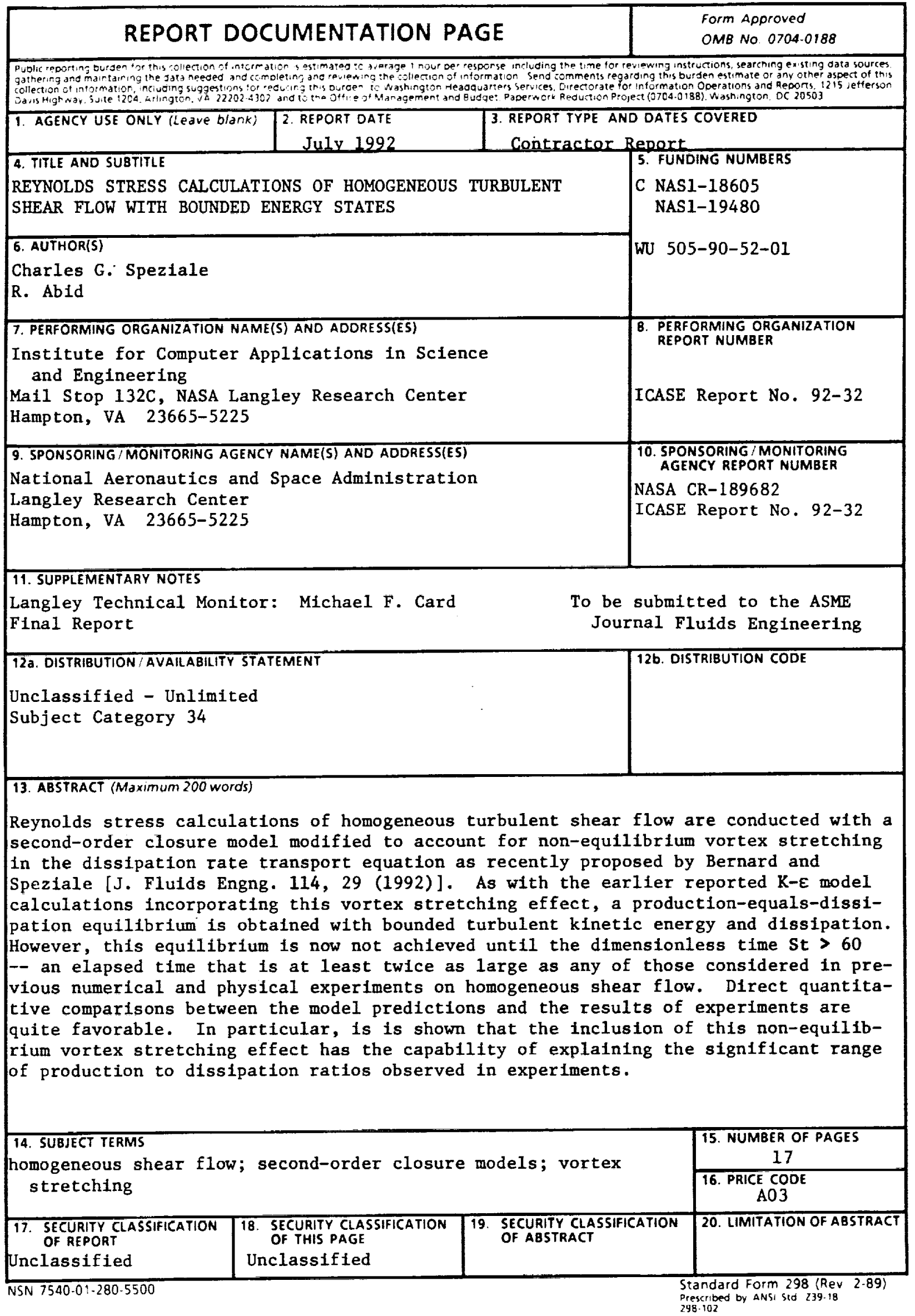

